TRANSACTIONS OF THE

AMERICAN MATHEMATICAL SOCIETY

Volume 355, Number 1, Pages 79-97

S 0002-9947(02)03140-9

Article electronically published on September 6, 2002

\title{
ABELIAN GROUPS WITH LAYERED TILES AND THE SUMSET PHENOMENON
}

\author{
RENLING JIN AND H. JEROME KEISLER
}

\begin{abstract}
We prove a generalization of the main theorem in Jin, The sumset phenomenon, about the sumset phenomenon in the setting of an abelian group with layered tiles of cell measures. Then we give some applications of the theorem for multi-dimensional cases of the sumset phenomenon. Several examples are given in order to show that the applications obtained are not vacuous and cannot be improved in various directions. We also give a new proof of Shnirel'man's theorem to illustrate a different approach (which uses the sumset phenomenon) to some combinatorial problems.
\end{abstract}

\section{INTRODUCTION}

A theorem in nonstandard analysis was proved in 4 in order to answer a question posed by Keisler and Leth in [5. This theorem implies many results in standard mathematics which reveal a general principle called the sumset phenomenon: if $A$ and $B$ are large in terms of "measure", then $A+B$ is not small in terms of "order-topology".

In $\S 1$ of this paper, we generalize [4. Theorem 1] to the setting of abelian groups with layered tiles of cell measures. The new generalization makes the applications in $\S 2$ to multi-dimensional cases easier. These applications show that the description of the sumset phenomenon needs to be adjusted with "measure" replaced by "product measure" and "order-topology" replaced by "product of order-topology". In $\S 3$, we construct several examples to show that the results about the sumset phenomenon in $\S 2$ and in [4] cannot be improved in various directions. For example, it was proven in [4 that if $A$ and $B$ are two subsets of the natural numbers with positive upper Banach density, then $A+B$ is piecewise syndetic, which means that there exists a fixed positive integer $k$ such that $A+B+\{0,1, \ldots, k\}$ is thick. It is natural to ask whether the least such $k$ is related to the upper Banach density of $A$ and the upper Banach density of $B$. One example constructed in $\S 3$ shows that $k$ is not directly related to these upper Banach densities. In $\S 4$, a new proof of Shnirel'man's theorem is given, which uses the idea of the sumset phenomenon rather than a finite combinatorial argument. In $\S 5$, two questions are raised.

Received by the editors October 10, 2001.

2000 Mathematics Subject Classification. Primary 20K99, 60B15, 22A05, 03H05; Secondary 11B05, 26E35, 28E05.

Key words and phrases. Abelian group, layered tiles of cell measures, the sumset phenomenon, upper Banach density.

The first author's research is supported in part by NSF grant DMS\#0070407.

The second author's research is supported in part by Vilas Trust Foundation. 


\section{Groups with Layered Tiles of Cell Measures}

Let $(G,+)$ be an abelian group. We often write $G$ for $(G,+)$. For any $A, B \subseteq G$ and $g \in G$, we write $A \pm B=\{a \pm b: a \in A$ and $b \in B\},-A=\{-a: a \in A\}$, $g \pm A=\{g\} \pm A$, and $A \pm g=A \pm\{g\}$. The lower case Greek letters $\alpha, \beta, \gamma, \delta, \varepsilon$ will always denote standard real numbers.

Definition 1.1. Given a set $C \subseteq G$ such that $0 \in C=-C$, a $C$-cell is a set $D$ of the form $C+g$ for some $g \in G$. $D$ is called the translation of $C$ by $g$, and $g$ is called the center of $D$.

A cell measure on $G$ is a pair $(C, \lambda)$ such that $0 \in C=-C, \lambda$ is a finitely additive measure on a Boolean algebra $\mathcal{B}$ of subsets of $G$ (called $\lambda$-measurable sets), $C \in \mathcal{B}, \lambda(C)=1$, and $\lambda$ is invariant under translations and reflections, that is, for each set $A \in \mathcal{B}$ and each $g \in G, A+g \in \mathcal{B},-A \in \mathcal{B}$, and $\lambda(A)=\lambda(A+g)=$ $\lambda(-A)$.

A cell measure tiling on $G$ is a triple $(C, \lambda, \mathcal{T})$ such that $(C, \lambda)$ is a cell measure, $\mathcal{T}$ is a pairwise disjoint family of $C$-cells such that the set of centers of cells in $\mathcal{T}$ is a subgroup $G_{\mathcal{T}}$ of $G$, and for each $\lambda$-measurable set $B$, the set

$$
\mathcal{T}(B)=\bigcup\{E \in \mathcal{T}: E \subseteq B\}
$$

is $\lambda$-measurable.

Here are some easy consequences of the definition.

Lemma 1.2. Suppose $(C, \lambda, \mathcal{T})$ is a cell measure tiling and $D$ is a $C$-cell.

(i) For each $g \in G, D+g$ and $-D$ are $C$-cells.

(ii) $D-D=C-C$.

(iii) $\lambda(D)=1$.

(iv) If $D \in \mathcal{T}$ and $g \in G_{\mathcal{T}}$, then $D+g \in \mathcal{T}$ and $-D \in \mathcal{T}$.

(v) If $A, B$ are $\lambda$-measurable, the set

$$
\mathcal{T}(A, B)=\bigcup\{E \in \mathcal{T}(B): E \cap A \neq \emptyset\}
$$

is $\lambda$-measurable, and

$$
A \cap \mathcal{T}(B) \subseteq \mathcal{T}(A, B) \subseteq \mathcal{T}(B) \subseteq B
$$

Proof. We have $D=C+h$ for some $h \in G$.

(i) $D+g=(C+h)+g=C+(h+g)$, and $-D=-(C+h)=-C-h=C-h$.

(ii) $D-D=(C+h)-(C+h)=(C-C)+(h-h)=C-C$.

(iii) Because $\lambda$ is invariant under translations.

(iv) Note that $h \in G_{\mathcal{T}}$ and use the proof of (i).

(v) $\mathcal{T}(A, B)=\mathcal{T}(B) \backslash \mathcal{T}(B \backslash A)$.

For a simple example of a cell measure tiling, let $d$ be a positive integer, let $G$ be the Euclidean vector space $\mathbb{R}^{d}$, and let $C$ be the open $d$-cube $(-1 / 2,1 / 2)^{d}$. Then the set of $C$-cells is the set of all open $d-$-cubes of side one. Now let $\lambda$ be the $d$-th product of Lebesgue measure and let $\mathcal{T}$ be the set of $C$-cells whose center is a $d$-tuple of integers. Then $(C, \lambda, \mathcal{T})$ is a cell measure tiling on $G$, and $G_{\mathcal{T}}$ is the subgroup $G_{\mathcal{T}}=\mathbb{Z}^{d}$. Further examples will appear later on in this paper.

In this paper $(P, \leqslant)$ always denotes a downward directed partial order with no least element. (A partial order is called downward directed if for any two elements $p$ and $q$, there is an $r$ with $r \leqslant p$ and $r \leqslant q$.) We say that each sufficiently small 
$s \in P$ has property $X$ if there exists $t \in P$ such that each $s<t$ has property $X$. Since $P$ is downward directed, if all sufficiently small $s \in P$ have property $X$ and all sufficiently small $s \in P$ have property $Y$, then all sufficiently small $s \in P$ have property $(X$ and $Y)$.

Here is the key definition in this paper.

Definition 1.3. An abelian group $G$ has layered tiles of cell measures of type $P$ if there is a real number $\delta \in(0,1)$ and a family of cell measure tilings $\left\{\left(C_{r}, \lambda_{r}, \mathcal{T}_{r}\right): r \in P\right\}$ such that the following hold for all $r \in P$.

(1) For each $s \in P, \lambda_{s}$ and $\lambda_{r}$ have the same measurable sets.

(2) If $s<r$, then $C_{s} \subseteq C_{r}$.

(3) There exists $s<r$ such that $C_{s}-C_{s} \subseteq C_{r}$.

(4) For every real $\varepsilon>0$, and for every sufficiently small $s \in P$ :

(a) for each $C_{r}$-cell $C, \lambda_{r}\left(C \backslash \mathcal{T}_{s}(C)\right)<\varepsilon$;

(b) for each $C_{r}-\operatorname{cell} C$ and measurable set $A, \lambda_{r}\left(A \cap \mathcal{T}_{s}(C)\right) \leqslant \alpha \lambda_{r}\left(\mathcal{I}_{s}(A, C)\right)$ where $\alpha=\sup \left\{\lambda_{s}(E \cap A): E\right.$ is a $C_{s}-$ cell $\}$;

(c) for all $C_{r}$-cells $C, D$ such that the center of $D$ is in $C, \lambda_{r}\left(\mathcal{T}_{s}(C \cap D)\right) \geqslant$ $\delta$.

Remarks. (1) The reader might want to keep a concrete example in mind in order to better grasp the idea. For example, one can think of $G$ as the group $\mathbb{R}^{2}$ with vector addition, $P$ as the set of all pairs of positive real numbers with the partial order

$$
(s, t) \geqslant\left(s^{\prime}, t^{\prime}\right) \text { iff } s \geqslant s^{\prime} \text { and } t \geqslant t^{\prime},
$$

$C_{(s, t)}$ as the open rectangle $(-s, s) \times(-t, t), \lambda_{(s, t)}=(4 s t)^{-1} \lambda$ where $\lambda$ is Lebesgue measure, and $\mathcal{T}_{(s, t)}$ as the set of $C_{(s, t)}-$ cells with centers $\{(2 s m, 2 t n): m, n \in \mathbb{Z}\}$.

(2) The word "layer" is used because one can think of the $C_{r}$-cells as a layer of cells at level $r$.

(3) Condition 4 (a) says that each $C_{r}$-cell is tiled by $C_{s}$-cells in the set $\mathcal{T}_{s}$ with an error less than $\varepsilon$.

(4) Condition 4 (b) says that if $A$ has density at most $\alpha$ within each tile, then $A$ has density at most $\alpha$ within the union of the tiles meeting $A$.

(5) Condition 4 (c) says that the union of the tiles inside $C \cap D$ has measure at least $\delta$. The constant $\delta$ is independent of $r$ and is a lower bound for the overlap between nearby cells. In the example in (1) above, one can take $\delta=1 / 5$.

(6) We define layered tiles of cell measures on an abelian group for convenience only. One can define the concept on a nonabelian group, but we have not yet seen any interesting applications of that case.

Let $\mathbb{C}=\left\{C_{r}: r \in P\right\}, \Lambda=\left\{\lambda_{r}: r \in P\right\}$, and $\mathbb{T}=\left\{\mathcal{T}_{r}: r \in P\right\}$. We write $(G,+, \mathbb{C}, \Lambda, \mathbb{T}, \delta)$ for a group with layered tiles of cell measures $\left(C_{r}, \lambda_{r}, \mathcal{T}_{r}\right)$ of type $P$ and overlap constant $\delta$. For the rest of this section, we fix such an abelian group $(G,+, \mathbb{C}, \Lambda, \mathbb{T}, \delta)$. We also let $\mathcal{C}_{r}$ be the set of all $C_{r}$-cells.

Definition 1.4. A set $A \subseteq G$ is called $\Lambda$-measurable if it is $\lambda_{r}$-measurable for some $r \in P$. For a $\Lambda$-measurable set $A \subseteq G$, the upper Banach density of $A$, $B D_{\Lambda}(A)$, is defined by

$$
B D_{\Lambda}(A)=\sup \left\{\lambda_{r}(A \cap C): r \in P, C \in \mathcal{C}_{r}\right\} .
$$

Definition 1.5. A set $A \subseteq G$ is called $\mathbb{C}$-nowhere dense if for every $r \in P$ and every $C \in \mathcal{C}_{r}$, there exists an $s<r$ and a $D \in \mathcal{C}_{s}$ such that $D \subseteq C$ and $D \cap A=\emptyset$. 
Note that the definition of $\mathbb{C}$-nowhere denseness does not mention any measure in $\Lambda$. We prove two more easy lemmas.

Lemma 1.6. Let $A$ be $\Lambda$-measurable and $g \in G$. Then

$$
B D_{\Lambda}(A)=B D_{\Lambda}(A+g)=B D_{\Lambda}(-A) .
$$

If $A$ is $\mathbb{C}-$ nowhere dense, then so are $A+g$ and $-A$.

Proof. This follows from the fact that each measure $\lambda_{r}$ is invariant under translations and reflections.

Lemma 1.7. Let $A$ be a $\Lambda$-measurable set with $B D_{\Lambda}(A)>\gamma$. Then for each sufficiently small $s \in P$, there is a $C_{s}$-cell $E$ such that $\lambda_{s}(A \cap E)>\gamma$.

Proof. By the definition of $B D_{\Lambda}$, one can find an $r \in P$ and a $C_{r}$-cell $C$ such that $\lambda_{r}(A \cap C)>\gamma$. Let

$$
\varepsilon=\lambda_{r}(A \cap C)-\gamma
$$

Each sufficiently small $s \in P$ satisfies Definition 1.3, part 4 with respect to $r$ and $\varepsilon$. Let

$$
\alpha=\sup \left\{\lambda_{s}(A \cap E): E \in \mathcal{C}_{s}\right\} .
$$

We have $\mathcal{T}_{s}(A, C) \subseteq C$; so $\lambda_{r}\left(\mathcal{T}_{s}(A, C)\right) \leqslant \lambda_{r}(C)=1$. Then by 4 (b),

$$
\lambda_{r}\left(A \cap \mathcal{T}_{s}(C)\right) \leqslant \alpha \lambda_{r}\left(\mathcal{T}_{s}(A, C)\right) \leqslant \alpha .
$$

Moreover,

$$
A \cap C \subseteq\left(A \cap \mathcal{T}_{s}(C)\right) \cup\left(C \backslash \mathcal{T}_{s}(C)\right)
$$

Therefore,

$$
\gamma+\varepsilon=\lambda_{r}(A \cap C) \leqslant \lambda_{r}\left(A \cap \mathcal{T}_{s}(C)\right)+\lambda_{r}\left(C \backslash \mathcal{T}_{s}(C)\right)<\alpha+\varepsilon .
$$

Then $\alpha>\gamma$; so there is a $C_{s}$-cell $E$ such that $\lambda_{s}(A \cap E)>\gamma$.

Next we state and prove the main theorem of the paper.

Theorem 1.8. Let $(G,+, \mathbb{C}, \Lambda, \mathbb{T}, \delta)$ be an abelian group with layered tiles of cell measures of type $P$. Let $A$ and $B$ be $\Lambda-$ measurable subsets of $G$. If $B D_{\Lambda}(A)>0$ and $B D_{\Lambda}(B)>0$, then $A+B$ is not $\mathbb{C}$-nowhere dense.

Proof. Assume that the theorem is not true. We will derive a contradiction. First we define two numbers $\alpha$ and $\beta$. Let

$$
\begin{aligned}
\alpha= & \sup \left\{B D_{\Lambda}(A): A \subseteq G \text { is } \Lambda\right. \text {-measurable and } \\
& \text { there is a } \Lambda \text {-measurable } B \subseteq G \text { with } B D_{\Lambda}(B)>0 \\
& \text { such that } A+B \text { is } \mathbb{C} \text {-nowhere dense }\} .
\end{aligned}
$$

Note that $\alpha$ is a positive real number by the assumption that the theorem is not true. Fix a positive real number

$$
\alpha_{0}<\frac{\alpha \delta}{3}
$$

and let

$$
\begin{aligned}
\beta= & \sup \left\{B D_{\Lambda}(B): B \subseteq G \text { is } \Lambda\right. \text {-measurable and } \\
& \text { there is a } \Lambda \text {-measurable } A \subseteq G \text { with } B D_{\Lambda}(A)>\alpha-\alpha_{0} \\
& \text { such that } A+B \text { is } \mathbb{C} \text {-nowhere dense }\} .
\end{aligned}
$$


It is easy to see that $\beta$ is positive and $\beta \leqslant \alpha$. Again fix a positive real number

$$
\beta_{0}<\frac{\beta \delta}{3} \text {. }
$$

We also fix two $\Lambda$-measurable sets $A, B \subseteq G$ such that

$$
B D_{\Lambda}(A)>\alpha-\alpha_{0}, \quad B D_{\Lambda}(B)>\beta-\beta_{0},
$$

and $A+B$ is $\mathbb{C}$-nowhere dense. It follows from the definitions of $\alpha$ and $\beta$ that $B D(A) \leqslant \alpha$ and $B D(B) \leqslant \beta$. By Lemma 1.7, for each sufficiently small $r \in P$, there are $C_{r}$-cells $C, D$ such that

$$
\lambda_{r}(A \cap C)>\alpha-\alpha_{0} \quad \text { and } \quad \lambda_{r}(B \cap D)>\beta-\beta_{0} .
$$

By Lemma 1.6 we may assume that $C=C_{r}$.

We have $D \subseteq C+D$ because $0 \in C$. Since $A+B$ is $\mathbb{C}$-nowhere dense, there exists $t<r$ and a $C_{t}-$ cell $F \subseteq D$ such that $F \cap(A+B)=\emptyset$. Let $g$ be the center of $D$ and let $f$ be the center of $F$, whence $D=C+g$ and $F=C_{t}+f$.

Let

$$
\varepsilon=\min \left(\frac{\alpha_{0}}{2}, \frac{\beta_{0}}{2}\right) .
$$

Each sufficiently small $s \in P$ satisfies Definition 1.3, part 4 for $\varepsilon, r$, and also satisfies $s<t$ and $C_{s}-C_{s} \subseteq C_{t}$. Then for each $E \in \mathcal{T}_{s}$, we have $E-E+f=C_{s}-C_{s}+f \subseteq$ $C_{t}+f=F$; so $E-E+f$ is disjoint from $A+B$. It follows that

$$
\mathcal{T}_{s}(A, C) \cap \mathcal{T}_{s}(f-B, f-D)=\emptyset,
$$

because if a $C_{s}-$ cell $E$ met both $A$ and $f-B$, then $f-E$ would meet $B$, and hence $E-E+f$ would meet $A+B$.

We have $f \in F \subseteq C+g$; so $f=c+g$ for some $c \in C$, and therefore $f-g \in C$. The $C_{r}$-cell with center $f-g$ is $f-(C+g)=f-D$. By 1.3 part 4 (c),

$$
\lambda_{r}\left(\mathcal{T}_{s}(C \cap(f-D))\right) \geq \delta .
$$

It follows that at least one of the sets

$$
\mathcal{T}_{s}(C \cap(f-D)) \backslash \mathcal{T}_{s}(A, C), \quad \mathcal{T}_{s}(C \cap(f-D)) \backslash \mathcal{T}_{s}(f-B, f-D)
$$

has $\lambda_{r}$-measure at least $\delta / 2$.

Suppose first that

$$
\lambda_{r}\left(\mathcal{T}_{s}(C \cap(f-D)) \backslash \mathcal{T}_{s}(A, C)\right) \geqslant \frac{\delta}{2} .
$$

Then

We have

$$
\lambda_{r}\left(\mathcal{T}_{s}(A, C)\right) \leqslant 1-\frac{\delta}{2}
$$

$$
\sup \left\{\lambda_{s}(A \cap E): E \in \mathcal{C}_{s}\right\} \leqslant B D_{\Lambda}(A) \leqslant \alpha .
$$

By part 4 (b) of Definition 1.3.

$$
\lambda_{r}\left(A \cap \mathcal{T}_{s}(C)\right) \leqslant \alpha \lambda_{r}\left(\mathcal{T}_{s}(A, C)\right) .
$$

Since

we have

$$
A \cap C \subseteq\left(A \cap \mathcal{T}_{s}(C)\right) \cup\left(C \backslash \mathcal{T}_{s}(C)\right),
$$

$$
\lambda_{r}(A \cap C) \leqslant \alpha \lambda_{r}\left(A \cap \mathcal{T}_{s}(C)\right)+\lambda_{r}\left(C \backslash \mathcal{T}_{s}(C)\right)<\alpha\left(1-\frac{\delta}{2}\right)+\varepsilon .
$$


Using (1), (3), and (4), we get the contradiction

$$
\alpha-\alpha_{0}<\alpha\left(1-\frac{\delta}{2}\right)+\varepsilon<\alpha\left(1-\frac{3 \alpha_{0}}{2 \alpha}\right)+\frac{\alpha_{0}}{2}=\alpha-\alpha_{0} .
$$

Now suppose that

$$
\lambda_{r}\left(\mathcal{T}_{s}(C \cap(f-D)) \backslash \mathcal{T}_{s}(f-B, f-D)\right) \geqslant \frac{\delta}{2} .
$$

Then

$$
\lambda_{r}\left(\mathcal{T}_{s}(f-B, f-D)\right) \leqslant 1-\frac{\delta}{2} .
$$

By repeating the above argument with $(f-B, f-D, \beta)$ instead of $(A, C, \alpha)$, we again get a contradiction. This completes the proof.

\section{The Sumset Phenomenon}

In this section we apply Theorem 1.8 to obtain new examples of the sumset phenomenon for multi-dimensional cases. Let $\mathbb{N}, \mathbb{Z}$, and $\mathbb{R}$ denote the set of all natural numbers including 0 , all integers, and all real numbers, respectively. From now on we fix a positive integer $d$ for the dimension of a space. In some applications we work within a countably saturated nonstandard universe ${ }^{*} \mathbf{V}$. The reader is assumed to have enough basic knowledge of nonstandard analysis to understand these applications. For more information on nonstandard analysis, consult [6], [7, or $\S 4.4$ of [2]. For any standard set $A$, the set ${ }^{*} A$ is always the nonstandard version of $A$ in ${ }^{*} \mathbf{V}$. For an abelian group $(G,+)$, we also use + for the addition on the product group $G^{d}$, i.e., $\left(f_{0}, f_{1}, \ldots, f_{d-1}\right)+\left(g_{0}, g_{1}, \ldots, g_{d-1}\right)=\left(f_{0}+g_{0}, f_{1}+g_{1}, \ldots, f_{d-1}+\right.$ $\left.g_{d-1}\right)$. In the first two applications, an interval means an interval of real numbers.

Application 2.1. Let $\nu$ be the Lebesgue measure on $\mathbb{R}$ and $\nu^{d}$ be the $d$-dimensional product of $\nu$ on $\mathbb{R}^{d}$. Let $A, B \subseteq \mathbb{R}^{d}$ be such that $\nu^{d}(A)>0$ and $\nu^{d}(B)>0$. Then $A+B$ contains a nonempty open ball.

Proof. It is sufficient to show that $A+B$ contains a nonempty $d$-dimensional open cube. Without loss of generality, we can assume that $A$ and $B$ are compact subsets of $\mathbb{R}^{d}$, because every set of positive $\nu^{d}$-measure contains a compact subset of positive $\nu^{d}$-measure. Since $A+B$ is closed, it is enough to prove that $A+B$ is not nowhere dense.

Let $G=\left(\mathbb{R}^{d},+\right)$. We now define the layered tiles of cell measures of type $P$. Let $P$ be the set of all positive real numbers with the natural order. For each $r \in P$, let $C_{r}=(-r, r)^{d}$. Then $\mathcal{C}_{r}$ is the set of all $d$-dimensional open cubes with sides of length $2 r$. Let $\lambda_{r}=(2 r)^{-d} \nu^{d}$, and let $\mathcal{T}_{r}$ be the set of all $C_{r}-$ cells with centers $(2 r m, 2 r n)$ where $m, n \in \mathbb{Z}$. Let $\mathbb{C}=\left\{C_{r}: r \in P\right\}, \Lambda=\left\{\lambda_{r}: r \in P\right\}$, and

$\mathbb{T}=\left\{\mathcal{T}_{r}: r \in P\right\}$, and let $\delta=2^{-d-1}$. It is clear that $\left(\mathbb{R}^{d},+, \mathbb{C}, \Lambda, \mathbb{T}, \delta\right)$ is a group with layered tiles of cell measures of type $P$, since each tiling $\mathcal{T}_{r}$ is countable. It is easy to see that $B D_{\Lambda}(A)>0$ and $B D_{\Lambda}(B)>0$. Therefore by Theorem 1.8, $A+B$ is not nowhere dense.

We now use the nonstandard reals to construct another group with layered tiles of cell measures. Let ${ }^{*} \mathbb{R}$ be the additive group of hyperreal numbers, which is the nonstandard version of the usual additive group of real numbers in ${ }^{*} \mathbf{V}$. 
A set $U$ of nonnegative elements of $* \mathbb{R}$ is called a cut in $* \mathbb{R}$ if:

(1) $0 \in U$;

(2) if $y \in U$ and $0 \leqslant x<y$, then $x \in U$; and

(3) $U+U \subseteq U$.

Note that $\{0\}$ and $\left\{r \in{ }^{*} \mathbb{R}: r \geqslant 0\right\}$ are cuts in ${ }^{*} \mathbb{R}$. Let $U$ be a cut in $* \mathbb{R}$ and $r$ be an element of ${ }^{*} \mathbb{R}$. We write $r>U$ if $r>s$ for every $s \in U$. A cut $U$ in ${ }^{*} \mathbb{R}$ is upper bounded if $r>U$ for some $r \in{ }^{*} \mathbb{R}$. For convenience we consider only upper bounded cuts in $* \mathbb{R}$ in the next application. Let $*\left(\nu^{d}\right)$ be the nonstandard version of $\nu^{d}$ and let $s t$ be the standard part map from $* \mathbb{R}$ to $\mathbb{R} \cup\{\infty\}$.

A cut in $* \mathbb{R}^{d}$ is a $d$-tuple $\mathbf{U}=\left(U_{0}, U_{1}, \ldots, U_{d-1}\right)$ of upper bounded cuts in $* \mathbb{R}$. Given a cut $\mathbf{U}$ and an element $\mathbf{r}=\left(r_{0}, \ldots, r_{d-1}\right)$ in ${ }^{*} \mathbb{R}^{d}, \mathbf{r}>\mathbf{U}$ means that $r_{j}>U_{j}$ for $j=0, \ldots, d-1$. A $\mathbf{U}$-box is a set of the form $\prod_{j=0}^{d-1}\left(a_{j}, b_{j}\right)$ where $b_{j}-a_{j}>U_{j}$ for each $j$. A set $A \subseteq{ }^{*} \mathbb{R}^{d}$ is called $\mathbf{U}$-nowhere dense if for every $\mathbf{U}$-box $C$, there is another $\mathbf{U}$-box $D$ such that $D \subseteq C$ and $D \cap A=\emptyset$.

Application 2.2. Let $A, B \subseteq{ }^{*} \mathbb{R}^{d}$ be internal sets and let $\mathbf{U}$ be a cut in ${ }^{*} \mathbb{R}^{d}$. If there exist two $\mathbf{U}$-boxes $C$ and $D$ such that

$$
s t\left(\frac{*\left(\nu^{d}\right)(A \cap C)}{*\left(\nu^{d}\right)(C)}\right)>0 \text { and } s t\left(\frac{*\left(\nu^{d}\right)(B \cap D)}{*\left(\nu^{d}\right)(D)}\right)>0,
$$

then $A+B$ is not $\mathbf{U}-$ nowhere dense.

Proof. Let $G={ }^{*} \mathbb{R}^{d}$. Let

$$
P=\left\{\mathbf{r} \in{ }^{*} \mathbb{R}^{d}: \mathbf{r}>\mathbf{U}\right\} .
$$

For any $\mathbf{r}, \mathbf{s} \in P$, define $\mathbf{r} \leqslant \mathbf{s}$ by $r_{j} \leqslant s_{j}$ for $j=0,1, \ldots, d-1$. Then $P$ is a downward directed partial ordering. For each $\mathbf{r} \in P$, let

$$
C_{\mathbf{r}}=\prod_{j=0}^{d-1}\left(-r_{j}, r_{j}\right)
$$

Let $\mathcal{B}$ be the Boolean algebra of all internal ${ }^{*}\left(\nu^{d}\right)$-measurable sets, and define $\lambda_{\mathbf{r}}$ by

$$
\lambda_{\mathbf{r}}(D)=\operatorname{st}\left(\frac{*\left(\nu^{d}\right)(D)}{*\left(\nu^{d}\right)\left(C_{\mathbf{r}}\right)}\right)
$$

for each $D \in \mathcal{B}$. Then each $\lambda_{\mathbf{r}}$ is a finitely additive measure on $\mathcal{B}$. Let the tiling $\mathcal{T}_{\mathbf{r}}$ be the set of all $C_{\mathbf{r}}$-cells with centers $\left(2 r_{0} z_{0}, \ldots, 2 r_{d-1} z_{d-1}\right)$ where $z_{0}, \ldots, z_{d-1} \in{ }^{*} \mathbb{Z}$.

Let $\mathbb{C}=\left\{C_{\mathbf{r}}: \mathbf{r} \in P\right\}, \Lambda=\left\{\lambda_{\mathbf{r}}: \mathbf{r} \in P\right\}, \mathbb{T}=\left\{\mathcal{T}_{\mathbf{r}}: \mathbf{r} \in P\right\}$, and $\delta=2^{-d-1}$. It is easy to see that $B D_{\Lambda}(A)>0$ and $B D_{\Lambda}(B)>0$. By Theorem 1.8 it suffices to verify that $\left({ }^{*} \mathbb{R}^{d},+, \mathbb{C}, \Lambda, \mathbb{T}, \delta\right)$ is an abelian group with layered tiles of cell measures of type $P$. Parts (1)-(3) of Definition 1.3 are clear. For part 4 , let $\varepsilon>0$ be real and $\mathbf{r} \in P$. For each $\mathbf{s} \in P$, the set $\mathcal{T}_{\mathbf{s}}$ is internal and ${ }^{*}$ countable, i.e., countable in the sense of the nonstandard universe. Take an integer $k$ such that $d / k<\varepsilon$. Then for any $\mathbf{s}<\mathbf{r} / k$ in $P$, part 4 of the definition follows by the transfer principle. In particular, for part 4 (b), consider any real $\beta>\alpha$ where $\alpha=\sup \left\{\lambda_{\mathbf{s}}(E \cap A): E \in \mathcal{C}_{\mathbf{s}}\right\}$. We have

$$
*\left(\nu^{d}\right)(E \cap A)<\beta \cdot *\left(\nu^{d}\right)\left(C_{\mathbf{s}}\right)
$$

for each $E \in \mathcal{C}_{\mathbf{s}}$, and therefore for any $\mathcal{C}_{\mathbf{r}}-$ cell $C$,

$$
\frac{*\left(\nu^{d}\right)\left(A \cap \mathcal{T}_{\mathbf{s}}(C)\right)}{*\left(\nu^{d}\right)\left(C_{\mathbf{r}}\right)}<\frac{\beta \cdot *\left(\nu^{d}\right)\left(\mathcal{T}_{\mathbf{s}}(A, C)\right)}{*\left(\nu^{d}\right)\left(C_{\mathbf{r}}\right)} .
$$


Taking standard parts,

$$
\lambda_{\mathbf{r}}\left(A \cap \mathcal{T}_{\mathbf{s}}(C)\right) \leqslant \beta \cdot \lambda_{\mathbf{r}}\left(\mathcal{T}_{\mathbf{s}}(A, C)\right) .
$$

Since this holds for all $\beta>\alpha$, we get the desired inequality

$$
\lambda_{\mathbf{r}}\left(A \cap \mathcal{T}_{\mathbf{s}}(C)\right) \leqslant \alpha \cdot \lambda_{\mathbf{r}}\left(\mathcal{T}_{\mathbf{s}}(A, C)\right) .
$$

For the rest of this section we deal with the abelian group $* \mathbb{Z}$, which is the nonstandard counterpart of the abelian group $\mathbb{Z}$ of integers under addition. From now on, by an interval we mean an interval in ${ }^{*} \mathbb{Z}$. An element $K \in{ }^{*} \mathbb{N} \backslash \mathbb{N}$ is called a hyperfinite integer. A set $S$ is called hyperfinite if $S$ is internal and the internal cardinality of $S$ is a hyperfinite integer (that is, $S$ is *finite but not finite). For a hyperfinite set $S$, let $\Sigma_{S}$ be the collection of all internal subsets of $S$. The set $\Sigma_{S}$ is also a hyperfinite set. Then one can define a normalized counting measure $\mu^{S}$ on $\Sigma_{S}$ (or simply on $S$ ) by putting $\mu^{S}(F)=|F| /|S|$ for each nonempty $F \in \Sigma_{S}$. Now $s t \circ \mu^{S}$ is a finitely additive standard probability measure defined on $\Sigma_{S}$.

A set $U \subseteq{ }^{*} \mathbb{N}$ is called a cut in ${ }^{*} \mathbb{N}$ if $U$ is an infinite initial segment of $* \mathbb{N}$ and $U+U \subseteq U$. Note that $U=\mathbb{N}$ is the smallest cut and ${ }^{*} \mathbb{N}$ is the largest cut. For convenience we always assume $U \neq{ }^{*} \mathbb{N}$. Given a positive integer $d$, a cut in ${ }^{*} \mathbb{N}^{d}$ is a $d$-tuple $\mathbf{U}=\left(U_{0}, U_{1}, \ldots, U_{d-1}\right)$ of cuts in ${ }^{*} \mathbb{N}$. As before, $U<r$ means that $u<r$ for all $u \in U$, and $\mathbf{U}<\mathbf{r}$ means that $U_{j}<r_{j}$ for $j=0, \ldots, d-1$. A product of intervals of the form $\prod_{j=0}^{d-1}\left(a_{j}, b_{j}\right) \subseteq{ }^{*} \mathbb{Z}^{d}$ is called a $\mathbf{U}-$ box if $b_{j}-a_{j}>U_{j}$ for each $j$. A set $A \subseteq{ }^{*} \mathbb{Z}^{d}$ is called $\mathbf{U}$-nowhere dense if for every $\mathbf{U}$-box $C$, there is a U-box $D$ such that $D \subseteq C \backslash A$.

In the one-dimensional case $d=1$, a $U$-box is just an interval $(a, b)$ such that $b-a>U$. In this case, it is sometimes convenient to borrow the gap function notation from [5]. Let $A \subseteq{ }^{*} \mathbb{Z}$ be internal and let $C$ be a $U$-box. Then the length of the largest gap of $A$ in $C$ is

$$
\operatorname{Gap}(A, C)=\max \{|D|: D \text { is a } U-\text { box and } D \subseteq C \backslash A\} .
$$

Thus an internal set $A$ is $U$-nowhere dense iff for every $U$-box $C, \operatorname{Gap}(A, C)>U$.

Application 2.3. Let $A, B \subseteq{ }^{*} \mathbb{Z}^{d}$ be internal sets and let $\mathbf{U}$ be a cut in ${ }^{*} \mathbb{N}^{d}$. If there are $\mathbf{U}$-boxes $C$ and $D$ such that $\operatorname{st}\left(\mu^{C}(A \cap C)\right)>0$ and $\operatorname{st}\left(\mu^{D}(B \cap D)\right)>0$, then $A+B$ is not $\mathbf{U}$-nowhere dense.

In the one-dimensional case, the conclusion says that for some $U$-box $E$,

$$
\operatorname{Gap}(A+B, E) \in U \text {. }
$$

Proof. Let $P=\left\{\mathbf{r} \in{ }^{*} \mathbb{N}^{d}: \mathbf{r}>\mathbf{U}\right\}$. We again define $\mathbf{r} \leqslant \mathbf{s}$ by $r_{j} \leqslant s_{j}$ for $j \in[0, d-1]$, and $P$ is downward directed. Let $G$ be the abelian group ${ }^{*} \mathbb{Z}^{d}$. For each $\mathbf{r} \in P$, let

$$
C_{\mathbf{r}}=\prod_{j=0}^{d-1}\left(-r_{j}, r_{j}\right)
$$

and let $\mathbb{C}=\left\{C_{\mathbf{r}}: \mathbf{r} \in P\right\}$. Let $\lambda_{\mathbf{r}}$ be the finitely additive measure defined on the class of all internal subsets $X$ of ${ }^{*} \mathbb{Z}^{d}$ by the rule

$$
\lambda_{\mathbf{r}}(X)=s t\left(\frac{|X|}{\left|C_{\mathbf{r}}\right|}\right)
$$


so that $\lambda_{\mathbf{r}}\left(C_{\mathbf{r}}\right)=1$. Let $\Lambda=\left\{\lambda_{\mathbf{r}}: \mathbf{r} \in P\right\}$. Let $\mathcal{T}_{\mathbf{r}}$ be the set of all $C_{\mathbf{r}}$-cells with centers of the form $\left(2 r_{0} z_{0}, \ldots, 2 r_{d-1} z_{d-1}\right)$ where $z_{0}, \ldots, z_{d-1} \in{ }^{*} \mathbb{Z}$, and let $\mathbb{T}=\left\{\mathcal{T}_{\mathbf{r}}: \mathbf{r} \in P\right\}$. Let $\delta=2^{-d-1}$

It is again easy to see that $B D_{\Lambda}(A)>0$ and $B D_{\Lambda}(B)>0$. Using the same argument as in Application 2.2, one can show that $\left({ }^{*} \mathbb{Z}^{d},+, \mathbb{C}, \Lambda, \mathbb{T}, \delta\right)$ is an abelian group with layered tiles of cell measures of type $P$. The desired result now follows from Theorem 1.8 .

Next we derive a variation of Application 2.3. Let $H$ be a hyperfinite integer, let ${ }^{*} \mathbb{Z}_{H}=[0, H-1]$, and let $\oplus$ be the usual addition modulo $H$. Then $\left({ }^{*} \mathbb{Z}_{H}, \oplus\right)$ is an abelian group. We also use $\oplus$ for the addition on the finite Cartesian product $\left({ }^{*} \mathbb{Z}_{H}\right)^{d}$ as usual. Let $\mu^{H}$ be the normalized counting measure on $\left({ }^{*} \mathbb{Z}_{H}\right)^{d}$. For each $j \in[0, d-1]$, let $U_{j} \subseteq{ }^{*} \mathbb{Z}_{H}$ be a cut in ${ }^{*} \mathbb{N}$. Note that a cut $U \subseteq{ }^{*} \mathbb{Z}_{H}$ is a relatively small subset of $* \mathbb{Z}_{H}$ because $U<H / m$ for any standard positive integer $m$. The definition of $\mathbf{U}$-nowhere denseness of a set $A \subseteq\left({ }^{*} \mathbb{Z}_{H}\right)^{d}$ is exactly the same as before.

Application 2.4. Let $A, B \subseteq\left({ }^{*} \mathbb{Z}_{H}\right)^{d}$ be two internal sets. If $s t\left(\mu^{H}(A)\right)>0$ and st $\left(\mu^{H}(B)\right)>0$, then $A \oplus B$ is not $\mathbf{U}$-nowhere dense.

Proof. By Application 2.3, it suffices to show that $A \oplus B$ is $\mathbf{U}$-nowhere dense iff $A+B$ is $\mathbf{U}$-nowhere dense. This follows from the fact that

$$
A \oplus B=\bigcup\left\{F_{h}-\mathbf{c}_{h}: h \in\{0,1\}^{[0, d-1]}\right\},
$$

where $F_{h}$ and $\mathbf{c}_{h}$ are defined by the following:

Let $E_{0}={ }^{*} \mathbb{Z}_{H}$ and $E_{1}={ }^{*} \mathbb{Z}_{2 H} \backslash{ }^{*} \mathbb{Z}_{H}$ and let $c_{0}=0$ and $c_{1}=H$. For each function $h$ from $[0, d-1]$ to $\{0,1\}$, define $F_{h}=\prod_{j=0}^{d-1}\left(E_{h(j)} \cap(A+B)\right)$ and $\mathbf{c}_{h}=\left(c_{h(o)}, c_{h(1)}, \ldots, c_{h(d-1)}\right)$.

Remark. The main theorem in [4] is a special case of Application 2.4 for dimension $d=1$.

The next result is in the standard world. Let $A$ be a subset of $\mathbb{N}^{d}$. The set $A \subseteq \mathbb{N}^{d}$ is called thick if for any $m$, there is an $\mathbf{r} \in \mathbb{N}^{d}$ such that $\mathbf{r}+[0, m]^{d} \subseteq A$. The set $A \subseteq \mathbb{N}^{d}$ is called piecewise syndetic if there is a $k \in \mathbb{N}$ such that $A+[0, k]^{d}$ is thick. The upper Banach density of $A, B D(A)$, is defined by

$$
B D(A)=\limsup _{k \rightarrow \infty}\left\{\frac{|A \cap E|}{|E|}: E=\mathbf{r}+[0, k]^{d} \text { for } \mathbf{r} \in \mathbb{N}^{d}\right\} .
$$

Application 2.5. Let $A, B \subseteq \mathbb{N}^{d}$. If $B D(A)>0$ and $B D(B)>0$, then $A+B$ is piecewise syndetic.

Proof. For each $j \in[0, d-1]$, let $U_{j}=\mathbb{N}$ and let $\mathbf{U}=\left(U_{0}, U_{1}, \ldots, U_{d-1}\right)$. By the transfer principle and $B D(A) \geqslant \alpha$, there is a hyperfinite integer $K$ and an $\mathbf{r} \in{ }^{*} \mathbb{N}^{d}$ such that

$$
\text { st }\left(\frac{\left|{ }^{*} A \cap\left(\mathbf{r}+[0, K]^{d}\right)\right|}{(K+1)^{d}}\right) \geqslant \alpha .
$$

Therefore $\operatorname{st}\left(\mu^{C}\left({ }^{*} A\right)\right) \geq \alpha>0$ where $C=[0, K]^{d}$. The same holds for ${ }^{*} B$. Thus by Application $\left[2.3{ }^{*} A+{ }^{*} B\right.$ is not $\mathbf{U}$-nowhere dense. It follows that there is a 
hyperfinite integer $K$ and an $\mathbf{r} \in{ }^{*} \mathbb{N}^{d}$ such that there is no $\mathbf{U}$-box $E \subseteq(\mathbf{r}+$ $\left.[0, K]^{d}\right) \backslash\left({ }^{*} A+{ }^{*} B\right)$. Define

$$
\left.k=\max \left\{m \in{ }^{*} \mathbb{N}:\left(\exists \mathbf{s} \in{ }^{*} \mathbb{N}^{d}\right) \mathbf{s}+[0, m-1]^{d} \subseteq\left(\mathbf{r}+[0, K]^{d}\right) \backslash\left({ }^{*} A+{ }^{*} B\right)\right)\right\} .
$$

Then $k$ must be finite. Therefore $\mathbf{r}+[0, K-k]^{d} \subseteq{ }^{*} A+{ }^{*} B-[0, k]^{d}$, and hence

$$
\mathbf{r}+[k, K]^{d} \subseteq{ }^{*} A+{ }^{*} B+[0, k]^{d} .
$$

This implies that $A+B$ is piecewise syndetic.

Remark. Application 2.5 is a multi-dimensional generalization of [4, Corollary 3], which is a complementary result to the following result in [3]: If $A \subseteq \mathbb{N}$ has positive upper Banach density, then $(A-A) \cap \mathbb{N}$ is syndetic.

When $A=B$, the result above can be improved. For simplicity, we consider only the one-dimensional case.

Application 2.6. Let $A \subseteq \mathbb{N}$ and $B D(A)=\alpha>0$. For any sequence of intervals $\left\langle\left[a_{i}, b_{i}\right]: i \in \mathbb{N}\right\rangle$ with $\lim _{i \rightarrow \infty}\left(b_{i}-a_{i}\right)=\infty$ such that

$$
\lim _{i \rightarrow \infty} \frac{\left|A \cap\left[a_{i}, b_{i}\right]\right|}{b_{i}-a_{i}+1}=\alpha
$$

and for any $\beta \in(0,2)$, there is a sequence of intervals $\left\langle\left[c_{i}, d_{i}\right]: i \in \mathbb{N}\right\rangle$ and there is a $k \in \mathbb{N}$ such that $\left[c_{i}, d_{i}\right] \subseteq\left[2 a_{i}, 2 b_{i}\right]$,

$$
\liminf _{i \rightarrow \infty} \frac{d_{i}-c_{i}}{b_{i}-a_{i}} \geqslant \beta
$$

and

$$
\bigcup_{i \in \mathbb{N}}\left[c_{i}, d_{i}\right] \subseteq A+A+[0, k]
$$

Proof. By the transfer principle, for every hyperfinite integer $K$, one has

$$
s t\left(\frac{\left|{ }^{*} A \cap\left[a_{K}, b_{K}\right]\right|}{b_{K}-a_{K}+1}\right)=\alpha .
$$

Therefore, by Application 2.3, * $A+{ }^{*} A$ is not $\mathbb{N}$-nowhere dense. Choose $\left[c_{K}^{\prime}, d_{K}^{\prime}\right] \subseteq$ $\left[2 a_{K}, 2 b_{K}\right]$ such that

$$
\frac{2-\beta}{3}<\frac{c_{K}^{\prime}-2 a_{K}}{b_{K}-a_{K}}<\frac{2-\beta}{2} \text { and } \frac{2-\beta}{3}<\frac{2 b_{K}-d_{K}^{\prime}}{b_{K}-a_{K}}<\frac{2-\beta}{2} .
$$

Then

$$
2=\frac{2 b_{K}-2 a_{K}}{b_{K}-a_{K}}=\frac{d_{K}^{\prime}-c_{K}^{\prime}}{b_{K}-a_{K}}+\frac{2 b_{K}-d_{K}^{\prime}}{b_{K}-a_{K}}+\frac{c_{K}^{\prime}-2 a_{K}}{b_{K}-a_{K}} \leqslant \frac{d_{K}^{\prime}-c_{K}^{\prime}}{b_{K}-a_{K}}+2-\beta .
$$

Hence one has

$$
\frac{d_{K}^{\prime}-c_{K}^{\prime}}{b_{K}-a_{K}} \geqslant \beta
$$

Claim 2.6.1. There exists a $k \in \mathbb{N}$ such that if $[x, y] \subseteq\left[c_{K}^{\prime}, d_{K}^{\prime}\right] \backslash\left({ }^{*} A+{ }^{*} A\right)$, then $y-x \leqslant k$. 
Proof of Claim 2.6.1. If the claim is not true, then there is an interval $[x, y] \subseteq$ $\left[2 a_{K}, 2 b_{K}\right] \backslash\left({ }^{*} A+{ }^{*} A\right)$ such that $y-x>\mathbb{N}$. Let $H$ be a hyperfinite integer such that $H^{2}<y-x$. For simplicity we assume that $H$ evenly divides $a_{K}, b_{K}$, and $x$. Let

$$
m=\max \left\{i \in{ }^{*} \mathbb{N}: a_{K}+i H \leqslant b_{K}\right\}=\frac{b_{K}-a_{K}}{H} .
$$

For each $i \in[0, m-1]$, let $E_{i}=\left[a_{K}+i H, a_{K}+(i+1) H-1\right]$. Whenever $(i+j) H=$ $x-2 a_{K}$, we have $E_{i}+E_{j} \subseteq[x, y]$, and therefore either

$$
{ }^{*} A \cap E_{i}=\emptyset \quad \text { or } \quad{ }^{*} A \cap E_{j}=\emptyset .
$$

Since

$$
x \geqslant c_{K}^{\prime}>\frac{2-\beta}{3}\left(b_{K}-a_{K}\right)+2 a_{K} \text { and } y \leqslant d_{K}^{\prime}<2 b_{K}-\frac{2-\beta}{3}\left(b_{K}-a_{K}\right),
$$

then

$$
\text { st }\left(\frac{x-2 a_{K}}{b_{K}-a_{K}}\right) \geqslant \frac{2-\beta}{3} \text { and } s t\left(\frac{2 b_{K}-y}{b_{K}-a_{K}}\right) \geqslant \frac{2-\beta}{3} \text {. }
$$

It follows that $[0, m-1]$ contains a subinterval $I$ such that $s t(|I| / m)>0$ and at least half of the points $i \in I$ are such that ${ }^{*} A \cap E_{i}=\emptyset$. Therefore, in view of equation (5), there exists an $i \in[0, m-1]$ such that

$$
s t\left(\frac{\left|{ }^{*} A \cap\left[a_{K}+i H, a_{K}+(i+1) H-1\right]\right|}{H}\right)>\alpha .
$$

By the transfer principle, one gets $B D(A)>\alpha$, a contradiction. $\quad \square$ (Claim 2.6.1.)

By the claim one can find a $k \in \mathbb{N}$ such that $\left[c_{K}^{\prime}+k, d_{K}^{\prime}+k\right] \subseteq{ }^{*} A+{ }^{*} A+[0, k]$. Let $k_{K}$ be the smallest of these $k^{\prime}$ 's. If the set $\left\{k_{K}: K\right.$ is hyperfinite $\}$ is unbounded in $\mathbb{N}$, then by countable saturation, there is a hyperfinite $k_{K}$ for some hyperfinite $K$. This contradicts that $k_{K}$ is always finite. Hence $k_{K}$ is upper bounded in $\mathbb{N}$. Let $k$ be an upper bound and let $c_{K}=c_{K}^{\prime}+k$ and $d_{K}=d_{K}^{\prime}+k$. Then

$$
\left[c_{K}, b_{K}\right] \subseteq\left({ }^{*} A+{ }^{*} A+[0, k]\right) \cap\left[2 a_{K}, 2 b_{K}\right]
$$

for every hyperfinite integer $K$. By the underspill principle, there is a sequence $\left\langle\left[c_{i}, d_{i}\right]: i>i_{0}\right\rangle$ that satisfies the conclusion of Application 2.6 for $i>i_{0}$. For $i \leqslant i_{0}$ simply let $\left[c_{i}, d_{i}\right]=\emptyset$; this completes the proof.

Next we derive a multi-dimensional generalization of [4, Corollary 2]. Let $\left(\mathbb{Z}^{d}\right)^{\mathbb{N}}$ denote the set of all functions from $\mathbb{N}$ to $\mathbb{Z}^{d}$. For any $f, g \in\left(\mathbb{Z}^{d}\right)^{\mathbb{N}}$, let $(f+$ $g)(m)=f(m)+g(m)$. A set of the form $S_{k}=\prod_{j=0}^{d-1}\left[a_{j}^{(k)}, b_{j}^{(k)}\right] \subseteq \mathbb{Z}^{d}$ is called a box. A sequence of boxes $\left\langle S_{k}: k \in \mathbb{N}\right\rangle$ is called large if for each $j \in[0, d-1]$, $\lim _{k \rightarrow \infty}\left(b_{j}^{(k)}-a_{j}^{(k)}\right)=\infty$.

Application 2.7. For each $k \in \mathbb{N}$, let $A_{k}, B_{k} \subseteq \mathbb{Z}^{d}$. If there are two large sequences of boxes $\left\langle S_{k}: k \in \mathbb{N}\right\rangle$ and $\left\langle T_{k}: k \in \mathbb{N}\right\rangle$ such that

$$
\liminf _{k \rightarrow \infty}\left(\frac{\left|A_{k} \cap S_{k}\right|}{\left|S_{k}\right|}\right)>0, \quad \liminf _{k \rightarrow \infty}\left(\frac{\left|B_{k} \cap T_{k}\right|}{\left|T_{k}\right|}\right)>0,
$$

then there is a large sequence of boxes $\left\langle Q_{k}: k \in \mathbb{N}\right\rangle$ such that

$$
\liminf _{k \rightarrow \infty}\left(\operatorname{Gap}\left(A_{k}+B_{k}, Q_{k}\right)\right)<\infty .
$$


Proof. Let $\mathcal{F}$ be a non-principal ultrafilter on $\mathbb{N}$. Suppose the nonstandard universe ${ }^{*} \mathbf{V}$ is obtained by taking the ultrapower of the standard universe $\mathbf{V}$ modulo $\mathcal{F}$. Then ${ }^{*} \mathbb{Z}=\mathbb{Z}^{\mathbb{N}} / \mathcal{F}$, the ultrapower of $\mathbb{Z}$ modulo $\mathcal{F}$. Every element in ${ }^{*} \mathbf{V}$ has the form $\left[\left\langle a_{k}: k \in \mathbb{N}\right\rangle\right]$, the equivalence class of $\left\langle a_{k}: k \in \mathbb{N}\right\rangle$ modulo $\mathcal{F}$, where $\left\langle a_{k}: k \in \mathbb{N}\right\rangle$ is a sequence in a set $S$ in $V$ called a representative of the class. Let

$$
U=\left\{x \in{ }^{*} \mathbb{N}: x<[\langle f(k): k \in \mathbb{N}\rangle] \text { for every } f \in \mathbb{N}^{\mathbb{N}} \text { with } \lim _{k \rightarrow \infty} f(k)=\infty\right\} .
$$

Then $U$ is a cut. Note that $U=\mathbb{N}$ iff $\mathcal{F}$ is a so-called p-point. Let $\mathbf{U}=$ $(U, U, \ldots, U)$. It is easy to verify that $\left[\left\langle P_{k}: k \in \mathbb{N}\right\rangle\right]$ is a $\mathbf{U}$-box iff it is represented by a large sequence of boxes. So $\left[\left\langle S_{k}: k \in \mathbb{N}\right\rangle\right]$ and $\left[\left\langle T_{k}: k \in \mathbb{N}\right\rangle\right]$ are $\mathbf{U}$-boxes and

$$
s t\left(\frac{\left|\left[\left\langle A_{k}: k \in \mathbb{N}\right\rangle\right] \cap\left[\left\langle S_{k}: k \in \mathbb{N}\right\rangle\right]\right|}{\left|\left[\left\langle S_{k}: k \in \mathbb{N}\right\rangle\right]\right|}\right)>0
$$

and

$$
s t\left(\frac{\left|\left[\left\langle B_{k}: k \in \mathbb{N}\right\rangle\right] \cap\left[\left\langle T_{k}: k \in \mathbb{N}\right\rangle\right]\right|}{\left|\left[\left\langle T_{k}: k \in \mathbb{N}\right\rangle\right]\right|}\right)>0 .
$$

Hence by Application 2.3 $\left[\left\langle A_{k}+B_{k}: k \in \mathbb{N}\right\rangle\right]$ is not $\mathbf{U}$-nowhere dense. Now the conclusion follows from the fact that a large sequence of boxes $\left\langle R_{k}: k \in \mathbb{N}\right\rangle$ with $R_{k} \cap\left(A_{k}+B_{k}\right)=\emptyset$ for all but finitely many $k \in \mathbb{N}$ generates a $\mathbf{U}$-box that is disjoint from $\left[\left\langle A_{k}+B_{k}: k \in \mathbb{N}\right\rangle\right]$.

\section{EXAMPLES}

In this section we give examples which show that the results obtained in $\S 2$ are not vacuous and cannot be improved in various directions. For convenience we only give examples in the one-dimensional case. The first four examples show that the results obtained in $\S 2$ are not vacuous. The first example is well known.

Example 3.1. For each $0<\alpha<1$, there is a compact subset $A$ of the unit interval of the real line such that $A$ is nowhere dense and the Lebesgue measure of $A$ is greater than $\alpha$.

The second example is from [5].

Example 3.2. Let $\alpha \in(0,1)$. For any hyperfinite integer $H$ and any cut $U \subseteq$ $[0, H-1]$, there is an internal set $A \subseteq[0, H-1]$ with $s t(|A| / H)>\alpha$ such that $A$ is $U$-nowhere dense.

Example 3.3. For any $\alpha \in(0,1)$, there is a set $A \subseteq \mathbb{N}$ such that $B D(A) \geqslant \alpha$ and $A$ is not piecewise syndetic.

Proof. Let $k_{0} \in \mathbb{N}$ be such that

$$
\sum_{k=k_{0}}^{\infty} \frac{k}{2^{k}}<1-\alpha
$$

For each $k \geqslant k_{0}$, let

$$
B_{k}=\bigcup_{m=k_{0}}^{k} \bigcup_{l=1}^{2^{k-m}}\left[l 2^{m}-m, l 2^{m}-1\right]
$$


Then $B_{k} \subseteq\left[0,2^{k}-1\right]$ and

$$
\frac{\left|B_{k}\right|}{2^{k}} \leqslant \frac{1}{2^{k}} \sum_{m=k_{0}}^{k} \sum_{l=1}^{2^{k-m}} m=\sum_{m=k_{0}}^{k} \frac{2^{k-m} m}{2^{k}}=\sum_{m=k_{0}}^{k} \frac{m}{2^{m}}<1-\alpha .
$$

Let $A_{k}=\left[0,2^{k}-1\right] \backslash B_{k}$ and let

$$
A=\bigcup_{k=k_{0}}^{\infty}\left(2^{k}-2^{k_{0}}+A_{k}\right)
$$

It is easy to check that $B D(A) \geqslant \alpha$ (in fact, the Shnirel'man density of $A$ is greater than or equal to $\alpha$ ). Since every interval of length $2^{k+1}$ contains a subinterval of length at least $k$, which is disjoint from $A, A$ is not piecewise syndetic.

Example 3.4. For any $\alpha \in(0,1)$, there is a sequence of sets $\left\langle A_{k} \subseteq\left[0,2^{k}-1\right]: k \in\right.$ $\mathbb{N}\rangle$ with

$$
\inf \left\{\frac{\left|A_{k}\right|}{2^{k}}: k \in \mathbb{N}\right\} \geqslant \alpha
$$

such that for every large sequence of intervals (one-dimensional boxes) $\left\langle\left[a_{k}, b_{k}\right]\right.$ : $k \in \mathbb{N}\rangle$, one has

$$
\lim _{k \rightarrow \infty} \operatorname{Gap}\left(A_{k},\left[a_{k}, b_{k}\right]\right)=\infty .
$$

Proof. Let $A_{k}$ be the same as in Example 3.3 for $k \geqslant k_{0}$ and let $A_{k}=\left[0,2^{k}-1\right]$ for $k<k_{0}$. It is easy to see that the sequence $\left\langle A_{k} \subseteq\left[0,2^{k}-1\right]: k \in \mathbb{N}\right\rangle$ has the required properties.

The last four examples show that the results in $\S 2$ cannot be improved in various directions.

Example 3.5. Let $H$ be a hyperfinite integer and let $U, V \subseteq[0, H-1]$ be two cuts with $U \backslash V \neq \emptyset$. For any $\alpha<1 / 2$, there exist two internal sets $A, B \subseteq[0, H-1]$ such that $s t(|A| / H) \geqslant \alpha, s t(|B| / H) \geqslant \alpha$, and for any interval $[a, b]$ with $b-a>U$,

$$
\operatorname{Gap}(A+B,[a, b])>V .
$$

Proof. Let $x \in U \backslash V$. Define $A$ and $B$ by the following:

$$
A=\bigcup\left\{[l x,[(l+\alpha) x]-1]: 0 \leqslant l \leqslant\left[\frac{H}{x}\right]-1\right\}
$$

and $B=A$. It is easy to check that $s t(|A| / H)=s t(|B| / H)=\alpha$. Since

$$
A+B \subseteq \bigcup\left\{[l x,[(l+2 \alpha) x]-1]: 0 \leqslant l \leqslant 2\left[\frac{H}{x}\right]-2\right\}
$$

and $2 \alpha<1$, for any interval $[a, b]$ with $b-a>U$, one has

$$
\operatorname{Gap}(A+B,[a, b]) \geqslant[(1-2 \alpha) x]-1>V .
$$

Remark. Using the same idea as in Example 3.2 and Example 3.6. one can modify the sets $A$ and $B$ in Example 3.5 to make them $U$-nowhere dense.

Look at Application 2.5 for the case of $d=1$. Given two sets $A, B \subseteq \mathbb{N}$, if $B D(A)>0$ and $B D(B)>0$, then there is an $m \in \mathbb{N}$ such that $A+B+[0, m]$ is thick. It is easy to see that if $B D(A)+B D(B)>1$, then $A+B$ is already thick. So $m=0$. Hence it is natural to guess that the number $m$ may be related to $B D(A)$ 
and $B D(B)$. The next example uses an idea similar to Example 3.5 to show that this is not the case.

Example 3.6. For any $\alpha$ and $\beta$ with $\alpha+\beta<1$ and for any $m \in \mathbb{N}$, there exist $A, B \subseteq \mathbb{N}$ such that $A$ and $B$ are not piecewise syndetic, $B D(A) \geqslant \alpha, B D(B) \geqslant \beta$, and $A+B+[0, m]$ is not thick.

Proof. Without loss of generality, we assume $m \geqslant 1$. Let $\varepsilon=1-\alpha-\beta$ and let

$$
\delta=\min \left\{\frac{\varepsilon}{4 \alpha+\varepsilon}, \frac{\varepsilon}{4 \beta+\varepsilon}\right\} .
$$

Let $A_{k}$ be the same sets constructed in Example 3.3 such that $\left|A_{k}\right| / 2^{k} \geqslant 1-\delta$ for each $k \geqslant k_{0}$, where $k_{0}$ can be chosen large enough so that $k_{0}>4 \mathrm{~m} / \varepsilon$. Let

$$
C=\bigcup_{k=k_{0}}^{\infty}\left(2^{k}-2_{0}^{k}+A_{k}\right) .
$$

Then $B D(C) \geqslant 1-\delta$ and $C$ is not piecewise syndetic. Now we construct $A$ and $B$ by:

$$
A=\bigcup_{a \in C}\left(k_{0} a+\left[0,\left[\left(\alpha+\frac{\varepsilon}{4}\right) k_{0}\right]\right]\right) \quad \text { and } \quad B=\bigcup_{a \in C}\left(k_{0} a+\left[0,\left[\left(\beta+\frac{\varepsilon}{4}\right) k_{0}\right]\right]\right) .
$$

Then

$$
B D(A) \geqslant B D(C)\left(\alpha+\frac{\varepsilon}{4}\right) \geqslant(1-\delta)\left(\alpha+\frac{\varepsilon}{4}\right) \geqslant\left(1-\frac{\varepsilon}{4 \alpha+\varepsilon}\right)\left(\frac{4 \alpha+\varepsilon}{4}\right)=\alpha .
$$

By the same reason, we have $B D(B) \geqslant \beta$. Since $C$ is not piecewise syndetic, then both $A$ and $B$ are not piecewise syndetic. For any $l \in \mathbb{N}$, since

$$
\left[l k_{0},(l+1) k_{0}-1\right] \cap(A+B) \subseteq\left[l k_{0}, l k_{0}+\left[(\alpha+\beta+\varepsilon / 2) k_{0}\right]\right],
$$

then

$$
\begin{aligned}
{\left[l k_{0},\right.} & \left.(l+1) k_{0}-1\right] \cap(A+B+[0, m]) \\
& \subseteq\left[l k_{0}, l k_{0}+\left[(\alpha+\beta+\varepsilon / 2) k_{0}\right]+m\right] \\
& \subseteq\left[l k_{0}, l k_{0}+\left[(\alpha+\beta+\varepsilon / 2) k_{0}\right]+\left[\varepsilon k_{0} / 4\right]\right] \\
& \subseteq\left[l k_{0}, l k_{0}+\left[(\alpha+\beta+3 \varepsilon / 4) k_{0}\right]\right] \\
& \subseteq\left[l k_{0}, l k_{0}+\left[(1-\varepsilon / 4) k_{0}\right]\right] \\
& \subseteq\left[l k_{0},(l+1) k_{0}-m-1\right] .
\end{aligned}
$$

Hence $A+B+[0, m]$ is not thick because any interval of length greater than $3 k_{0}$ contains an interval of the form $\left[l k_{0},(l+1) k_{0}-1\right]$, which contains a subinterval $\left[(l+1) k_{0}-m,(l+1) k_{0}-1\right]$ disjoint from $A+B+[0, m]$.

Assume $d=1$ as in Application 2.7 The conclusion there is

$$
\liminf _{k \rightarrow \infty}\left(\operatorname{Gap}\left(A_{k}+B_{k}, Q_{k}\right)\right)<\infty .
$$

Is it possible that the conclusion can be strengthened to

$$
\limsup _{k \rightarrow \infty}\left(\operatorname{Gap}\left(A_{k}+B_{k}, Q_{k}\right)\right)<\infty ?
$$

The next example shows that it cannot. 
Example 3.7. For any nonnegative real numbers $\alpha$ and $\beta$ such that $\alpha+\beta<1$, there exist two sequences of sets $\left\langle A_{k} \subseteq\left[0,2^{k}-1\right]: k \in \mathbb{N}\right\rangle$ and $\left\langle B_{k} \subseteq\left[0,2^{k}-1\right]: k \in \mathbb{N}\right\rangle$ with

$$
\inf \left\{\frac{\left|A_{k}\right|}{2^{k}}: k \in \mathbb{N}\right\} \geqslant \alpha \text { and } \quad \inf \left\{\frac{\left|B_{k}\right|}{2^{k}}: k \in \mathbb{N}\right\} \geqslant \beta
$$

such that for any large sequence of intervals $\left\langle\left[a_{k}, b_{k}\right]: k \in \mathbb{N}\right\rangle$ the set

$$
\left\{\operatorname{Gap}\left(A_{k}+B_{k},\left[a_{k}, b_{k}\right]\right): k \in \mathbb{N}\right\}
$$

is unbounded in $\mathbb{N}$.

Proof. Choose a non-principal ultrafilter $\mathcal{F}$ on $\mathbb{N}$ that is not a p-point. Let the nonstandard universe and the cut $U$ be the same as in Application 2.7. Let $V=\mathbb{N}$. Since $\mathcal{F}$ is not a p-point, then $U \backslash V \neq \emptyset$. Let $H=\left[\left\langle 2^{k}: k \in \mathbb{N}\right\rangle\right]$. Then $U, V \subseteq[0, H-1]$. By Example 3.5 there exist internal sets $A=\left[\left\langle A_{k}: k \in \mathbb{N}\right\rangle\right]$ and $B=\left[\left\langle B_{k}: k \in \mathbb{N}\right\rangle\right]$ in $[0, H-1]$ such that $s t(|A| / H) \geqslant \alpha, \operatorname{st}(|B| / H) \geqslant \beta$, and for any interval $[a, b] \subseteq[0, H-1]$ of length greater than $U$,

$$
\operatorname{Gap}(A+B,[a, b])>V .
$$

Let

$$
X=\left\{k \in \mathbb{N}: \frac{\left|A_{k}\right|}{2^{k}} \geqslant \alpha \text { and } \frac{\left|B_{k}\right|}{2^{k}} \geqslant \beta\right\} .
$$

So $X \in \mathcal{F}$. For each $k \in X$, let $A_{k}$ and $B_{k}$ be unchanged and for each $k \notin X$, redefine $A_{k}=B_{k}=\left[0,2^{k}-1\right]$. If $\left\langle\left[a_{k}, b_{k}\right] \subseteq\left[0,2\left(2^{k}-1\right)\right]: k \in \mathbb{N}\right\rangle$ is a large sequence of intervals, then the interval $\left[\left[\left\langle a_{k}: k \in \mathbb{N}\right\rangle\right],\left[\left\langle b_{k}: k \in \mathbb{N}\right\rangle\right]\right] \subseteq[0,2 H-2]$ has length greater than $U$. Hence

$$
\left[\left\langle\operatorname{Gap}\left(A_{k}+B_{k},\left[a_{k}, b_{k}\right]\right): k \in \mathbb{N}\right\rangle\right]>V=\mathbb{N} .
$$

This implies that $\left\{\operatorname{Gap}\left(A_{k}+B_{k},\left[a_{k}, b_{k}\right]\right): k \in \mathbb{N}\right\}$ is not bounded in $\mathbb{N}$.

The last example shows that one cannot replace Lebesgue measure by outer Lebesgue measure in Application 2.1. We use [0,1] for the unit interval of real numbers in the next example.

Example 3.8. There is an $A \subseteq[0,1]$ such that the outer measure of $A$ is 1 and $A+A$ does not contain a nonempty open interval of real numbers.

Proof. In the proof below, we use $\alpha$ and $\beta$ for ordinals. Let $\mathfrak{c}=2^{\aleph_{0}}$ be the cardinality of the continuum and let $\left\{X_{\alpha}: \alpha<\mathfrak{c}\right\}$ be an enumeration of all uncountable closed subsets of $[0,1]$. View $\mathbb{R}$ as a linear space over $\mathbb{Q}$, the rational field. For any $Y \subseteq \mathbb{R}$, let $\langle Y\rangle$ denote the subspace generated by $Y$. We now construct two sequences of subsets of $[0,1], A_{0} \subseteq A_{1} \subseteq A_{2} \subseteq \cdots$ and $B_{0} \subseteq B_{1} \subseteq B_{2} \subseteq \cdots$, inductively such that for each $\alpha<\mathfrak{c}$,

(1) $\left\langle A_{\alpha}\right\rangle \cap[0,1]=A_{\alpha}$ and $\left\langle B_{\alpha}\right\rangle \cap[0,1]=B_{\alpha}$,

(2) $\left\langle A_{\alpha}\right\rangle \cap\left\langle B_{\alpha}\right\rangle=\{0\}$,

(3) the cardinality of $A_{\alpha} \cup B_{\alpha}<\mathfrak{c}$,

(4) $A_{\alpha} \cap X_{\alpha} \neq \emptyset$ and $B_{\alpha} \cap X_{\alpha} \neq \emptyset$.

Suppose $A_{\beta}$ and $B_{\beta}$ are obtained so that $1-4$ above are true for every $\beta<\alpha$. By 3, $\left|X_{\alpha} \backslash \bigcup_{\beta<\alpha}\left(A_{\beta} \cup B_{\beta}\right)\right|=\mathfrak{c}$. By a cardinality argument, one can choose $x, y \in X_{\alpha} \backslash \bigcup_{\beta<\alpha}\left(A_{\beta} \cup B_{\beta}\right)$ such that

$$
\left\langle\bigcup_{\beta<\alpha} A_{\beta} \cup\{x\}\right\rangle \cap\left\langle\bigcup_{\beta<\alpha} B_{\beta} \cup\{y\}\right\rangle=\{0\} .
$$


Now let

$$
A_{\alpha}=\left\langle\bigcup_{\beta<\alpha} A_{\beta} \cup\{x\}\right\rangle \cap[0,1] \quad \text { and } \quad B_{\alpha}=\left\langle\bigcup_{\beta<\alpha} B_{\beta} \cup\{y\}\right\rangle \cap[0,1] .
$$

It is clear that 1-4 are true for $A_{\alpha}$ and $B_{\alpha}$. Finally, let

$$
A=\bigcup_{\alpha<\mathfrak{c}} A_{\alpha} \quad \text { and } \quad B=\bigcup_{\alpha<\mathfrak{c}} B_{\alpha} .
$$

We now check that $A$ is the set we want. $A$ has outer measure 1 because of 4 . $A+A$ does not contain any nonempty open interval because of 2 and 4 .

\section{A New Proof of Shnirel'man's Theorem}

This section offers neither an "easier" nor a "better" proof of Shnirel'man's theorem. It merely offers a "different" proof. The purpose of this section is to demonstrate the potential of the sumset phenomenon in dealing with combinatorial problems involving asymptotic arguments. A conventional proof can be found in [8].

For a set $A \subseteq \mathbb{N}$, the Shnirel'man density of $A$ is defined by the following:

$$
\sigma(A)=\inf _{k \geqslant 1} \frac{|A \cap[1, k]|}{k} .
$$

Note that if $\sigma(A) \geqslant \alpha$, then by the transfer principle,

$$
\text { st }\left(\frac{\left|{ }^{*} A \cap[0, H-1]\right|}{H}\right) \geqslant \alpha
$$

for every hyperfinite integer $H$. Note also that if $\sigma(A)>0$, then $1 \in A$. For any set $A \subseteq \mathbb{N}$ and for any $h \in \mathbb{N}$, let

$$
h A=\underbrace{A+A+\cdots+A}_{h}
$$

if $h>0$ and let $h A=\emptyset$ if $h=0$. If $U$ is a cut and $x \in{ }^{*} \mathbb{N} \backslash U$, then

$$
x U=\left\{y \in{ }^{*} \mathbb{N}: y<x u \text { for some } u \in U\right\}
$$

and

$$
x / U=\left\{y \in{ }^{*} \mathbb{N}: y<x / u \text { for every } u \in U\right\}
$$

are cuts. Note that $x \mathbb{N}$ is the smallest cut containing $x$ and $x / \mathbb{N}$ is the largest cut not containing $x$.

Theorem 4.1 (Shnirel'man). For any set $A \subseteq \mathbb{N}$, if $0 \in A$ and $\sigma(A)>0$, then there is an $h \in \mathbb{N}$ such that $h A=\mathbb{N}$.

Proof. We suppose the theorem is not true and derive a contradiction. Since $h^{*} A$ $\neq{ }^{*} \mathbb{N}$ for every $h \in \mathbb{N}$, then $\bigcup_{h \in \mathbb{N}}\left(h^{*} A\right) \neq{ }^{*} \mathbb{N}$ by countable saturation. Let $H \in{ }^{*} \mathbb{N} \backslash \bigcup_{h \in \mathbb{N}}\left(h^{*} A\right)$.

Claim 4.1.1. Let $K$ be a hyperfinite integer and let $[a, b] \subseteq[0, K-1]$ be such that $b-a>K / n$ for some $n \in \mathbb{N}$. Then $[a, b] \cap\left(h^{*} A\right) \neq \emptyset$ for some $h \in \mathbb{N}$. 
Proof of Claim 4.1.1. Since

$$
s t\left(\frac{{ }^{*} A \cap[1,[K / n]] \mid}{[K / n]}\right)>0
$$

then

$$
{ }^{*} A \cap([0,[K / n]] \backslash K / \mathbb{N}) \neq \emptyset .
$$

Let $x \in{ }^{*} A$ be such that $K / \mathbb{N}<x<K / n$. Then $x<b-a$, and it is easy to see that there is a finite $h \in \mathbb{N}$ such that $h x \in[a, b]$. So $h x \in h^{*} A \cap[a, b] . \quad \square$ (Claim 4.1.1)

Claim 4.1.2. Suppose that $\operatorname{Gap}\left(2^{*} A,[a, b]\right) \leq n(d-c)$ and $[c, d] \subseteq h^{*} A$. Then

$$
[a+n d, b+n c] \subseteq(2+n h)^{*} A .
$$

Proof of Claim 4.1.2. Let $y=\min \left(2^{*} A \cap[a, b]\right)$ and $z=\max \left(2^{*} A \cap[a, b]\right)$. Then

$$
[y+n c, z+n d] \subseteq 2^{*} A+[n c, n d] \subseteq(2+n h)^{*} A .
$$

Moreover, $y \leqslant a+n(d-c), b-n(d-c) \leqslant z ;$ so $[a+n d, b+n c] \subseteq[y+n c, z+n d]$. $\square($ Claim 4.1.2)

Let

$$
\mathcal{V}=\{U: U \text { is a cut, } U<H, \text { and } H / \mathbb{N} \neq U\},
$$

and let

$$
\mathcal{U}=\left\{U \in \mathcal{V}:(\exists[a, b] \subseteq H / \mathbb{N})(\exists h \in \mathbb{N})[a, b] \subseteq h^{*} A \text { and } U<b-a\right\} .
$$

Note that $\mathcal{U}$ is downward closed, i.e., $U \subseteq V$ and $V \in \mathcal{U}$ imply $U \in \mathcal{U}$.

Claim 4.1.3. $\mathbb{N} \in \mathcal{U}$.

Proof of Claim 4.1.3. For each $x \in H / \mathbb{N}$, st $\left(\left.\right|^{*} A \cap[0, x] \mid / x\right) \geqslant \sigma(A)>0$. By Application [2.3, $\left.\left(2^{*} A\right) \cap[0,2 x]\right)$ is not $\mathbb{N}$-nowhere dense. Therefore there exists an interval $[a, b] \subseteq[0,2 x]$ for some $x \in H / \mathbb{N}$ such that $b-a>\mathbb{N}$ and $\operatorname{Gap}\left(2^{*} A,[a, b]\right)=m \in \mathbb{N}$. Since $[0,1] \subseteq{ }^{*} A$, it follows from Claim 4.1.2 that

$$
[a+m, b] \subseteq(2+m)^{*} A .
$$

$\square($ Claim 4.1.3)

Claim 4.1.4. $\mathcal{U}=\mathcal{V}$.

Proof of Claim 4.1.4. Suppose $\mathcal{U} \neq \mathcal{V}$. Let $\bar{V}=\bigcap(\mathcal{V} \backslash \mathcal{U})$. Then $\bar{V}$ is a cut.

Let $x \in H / \mathbb{N} \backslash \bar{V}$. By Application 2.3 , there is an interval $[a, b] \subseteq[0,2 x]$ with $b-a>\bar{V}$ such that $\operatorname{Gap}\left(2^{*} A,[a, b]\right)=l \in \bar{V}$.

Case 1. $l$ is finite. Then by Claim 4.1.2,

$$
[a+l, b] \subseteq 2^{*} A+[0, l] \subseteq(2+l)^{*} A .
$$

Therefore, in this case, we have $\bar{V} \in \mathcal{U}$.

Case 2. $l$ is hyperfinite. Let $U=l / \mathbb{N}$. Then $U<l \in \bar{V}$, and so $U \in \mathcal{U}$. Hence there exist $h^{\prime} \in \mathbb{N}$ and an interval $[c, d] \subseteq H / \mathbb{N}$ such that $U<d-c \in \bar{V}$ and $[c, d] \subseteq h^{* *} A$. Let $n \in \mathbb{N}$ be such that $d-c>l / n$. By Claim 4.1.2 again,

$$
[a+n d, b+n c] \subseteq\left(2+n h^{\prime}\right){ }^{*} A .
$$

Moreover, $(b+n c)-(a+n d)=(b-a)-n(d-c)>\bar{V}$. Thus, in this case, we also have $\bar{V} \in \mathcal{U}$.

By the definition of $\mathcal{U}$, there exist $h \in \mathbb{N}$ and $[a, b] \subseteq H / \mathbb{N}$ such that $b-a>\bar{V}$ and $[a, b] \subseteq h^{*} A$. Let $V=(b-a) / \mathbb{N}$. Then $V \supseteq \bar{V}$ because $V$ is the largest cut 
not containing $b-a$. Also, $V \in \mathcal{U}$ by the definition of $\mathcal{U}$. So $(b-a) \mathbb{N} \subseteq \bar{V}$ because $(b-a) \mathbb{N}$ is the smallest cut containing $b-a$. This contradicts $(b-a) / \mathbb{N} \supseteq \bar{V}$ and completes the proof that $\mathcal{U}=\mathcal{V} . \quad \square($ Claim 4.1.4)

Claim 4.1.5. There exist $h \in \mathbb{N}$ and $[a, b] \subseteq[0, H-1]$ such that $b-a>H / \mathbb{N}$ and $[a, b] \subseteq h^{*} A$.

Proof of Claim 4.1.5. By Application 2.3, there is an interval

$$
[c, d] \subseteq[0,[(H-1) / 2]]
$$

such that $d-c>H / \mathbb{N}$ and

$$
\operatorname{Gap}\left(2^{*} A,[c, d]\right)=l<H / \mathbb{N} .
$$

Let $U=l \mathbb{N}$. Then $U \neq H / \mathbb{N}$ by countable saturation. So by Claim 4.1.4, there exist $h^{\prime} \in \mathbb{N}$ and $[e, f] \subseteq H / \mathbb{N}$ such that $f-e>U$ and $[e, f] \subseteq h^{\prime *} A$. Let $[a, b]=[c+f, d+e]$. Then by Claim 4.1.2,

$$
[a, b] \subseteq\left(2+h^{\prime}\right)^{*} A .
$$

It is obvious that $0 \leqslant a$ and $b<[(H-1) / 2]+[(H-1) / 2] \leqslant H-1 . \quad \square($ Claim 4.1.5)

Let $[a, b] \subseteq[0, H-1]$ and $h \in \mathbb{N}$ be obtained according to Claim 4.1.5. By Claim 4.1.1, there is an $h^{\prime} \in \mathbb{N}$ such that $[H-b, H-a] \cap\left(h^{\prime *} A\right) \neq \emptyset$. Let

$$
r \in[H-b, H-a] \cap\left(h^{\prime *} A\right) .
$$

Then

$$
H \in[a+r, b+r]=[a, b]+r \subseteq\left(h^{*} A\right)+\left(h^{\prime *} A\right)=\left(h+h^{\prime}\right)^{*} A .
$$

This contradicts the choice of $H$ and completes the proof.

\section{Questions}

In this section we pose two open questions.

Let $H$ be a hyperfinite integer, and let $I(H)$ be the set of all internal subsets of $[0, H-1] \cap * \mathbb{N}$. For $A \in I(H)$ and $m \in * \mathbb{N}$, let

$$
g(A, m)=\min \{\operatorname{Gap}(A,[a, b]): b-a+1=m\} .
$$

It is easy to see that if $m>0$ and $A \neq \emptyset$, then $g(A, m)<m / 2$. One can define an internal decreasing sequence

$$
S(H, A)=\left\langle m_{i}: 0 \leqslant i \leqslant k\right\rangle
$$

such that $m_{0}=H, m_{i+1}=g\left(A, m_{i}\right)>0$ for $i \in[0, k-1]$, and $m_{k}=0$. The number $k$ is called the length of the sequence $S(H, A)$ and is denoted by $\operatorname{len}(S(H, A)$ ).

If $U \subseteq[0, H-1]$ is a cut and $A$ is $U$-nowhere dense, then the length of the sequence $S(H, A)$ is a hyperfinite integer. On the other hand, if the length of the sequence $S(H, A)$ is hyperfinite, then

$$
U=\bigcap_{i \in \mathbb{N}}\left[0, m_{i}\right]
$$

is a cut and $A$ is $U$-nowhere dense. Therefore by Application [2.3, if $A, B \in I(H)$ and

$$
s t\left(\frac{|A \cap[0, H-1]|}{H}\right)>0 \text { and } \operatorname{st}\left(\frac{|B \cap[0, H-1]|}{H}\right)>0 \text {, }
$$


then the length of the sequence $S(H, A+B)$ is finite. Given positive standard real numbers $\alpha$ and $\beta$ such that $\alpha+\beta<1$, let

$$
L(H, \alpha, \beta)=\left\{\operatorname{len}(S(H, A+B)): A, B \in I(H), \frac{|A|}{H} \geqslant \alpha, \text { and } \frac{|B|}{H} \geqslant \beta\right\} .
$$

Then $L(H, \alpha, \beta)$ is an internal subset of $\mathbb{N}$, and hence is upper bounded in $\mathbb{N}$.

Question 5.1. Given $H, \alpha, \beta$, what is the least upper bound of $L(H, \alpha, \beta)$ ?

Let $(G, \cdot)$ be a countable group not necessarily abelian. For $g \in G$ and $A \subseteq G$, let $g A=\{g a: a \in A\}$ and $A g=\{a g: a \in A\}$. We would like to define upper

Banach density and syndeticity of a subset of $G$ following the ideas in [1]. For convenience we consider only groups rather than semigroups.

A sequence $\left\langle A_{k}: k \in \mathbb{N}\right\rangle$ of subsets of $G$ is called a left Følner sequence if for every $g \in G$,

$$
\lim _{k \rightarrow \infty} \frac{\left|g A_{k} \Delta A_{k}\right|}{\left|A_{k}\right|}=0
$$

where $\Delta$ is the symmetric difference. If $G$ admits left $F \varnothing$ lner sequences, then for each $A \subseteq G$, one can define the upper Banach density of $A$ by the following:

$$
B D(A)=\sup \left\{\limsup _{k \rightarrow \infty} \frac{\left|A \cap A_{k}\right|}{\left|A_{k}\right|}:\left\langle A_{k}: k \in \mathbb{N}\right\rangle \text { is a left Følner sequence }\right\} .
$$

A set $A \subseteq G$ is called right thick if for every finite $F \subseteq G$, there is a $g \in G$ such that $F g \subseteq A$. A set $A \subseteq G$ is called right piecewise syndetic if there is a finite subset $F$ of $G$ such that $\bigcup_{f \in F} f A$ is right thick.

Question 5.2. Let $G$ be a countable group admitting left Følner sequences. Let $A, B \subseteq G$. Is $A \cdot B=\{a b: a \in A, b \in B\}$ always right piecewise syndetic whenever $B D(A)>0$ and $B D(B)>0$ ?

\section{REFERENCES}

[1] Bergelson, V., Hindman, N., and McCutcheon, R., Notions of size and combinatorial properties of quotient sets in semigroups, Topology Proceedings, 23 (1998), pp. 23-60. MR 2001a:20114

[2] Chang, C. C. and Keisler, H. J., Model Theory, third edition, North-Holland, 1990. MR 91c:03026

[3] Furstenberg, H., Recurrence in Ergodic Theory and Combinatorial Number Theory, Princeton University Press, 1981. MR 82j:28010

[4] Jin, Renling, The Sumset phenomenon, Proc. Amer. Math. Soc., to appear.

[5] Keisler, H. Jerome and Leth, Steven C., Meager Sets on the Hyperfinite Time Line, The Journal of Symbolic Logic, 56 (1991), pp. 71-102. MR 93a:03074

[6] Henson, C. W., Foundations of nonstandard analysis: A gentle introduction to nonstandard extensions, in Nonstandard Analysis: Theory and Applications, ed. by N. J. Cutland, C. W. Henson, and L. Arkeryd, Kluwer Academic Publishers, Dordrecht, 1997. MR 99i:03085

[7] Lindstrøm, T., An invitation to nonstandard analysis, in Nonstandard Analysis and its Applications, ed. by N. Cutland, Cambridge University Press, Cambridge, 1988. CMP 21:05

[8] Nathanson, M. B., Additive Number Theory: The Classical Bases, Springer-Verlag, New York, 1996. 29424

Department of Mathematics, College of Charleston, Charleston, South Carollina

E-mail address: jinr@cofc.edu

Department of Mathematics, University of Wisconsin, Madison, Wisconsin 53706

E-mail address: keisler@math.wisc.edu 